\title{
Direction of Estimation Approach for Collaborative Localization in Wireless Sensor Networks
}

\author{
A. I. Alhasanat, B. S. Sharif, C. C. Tsimenidis, and S. Boussakta \\ School of Electrical, Electronic and Computer Engineering / Newcastle University \\ Newcastle Upon Tyne, United Kingdom, NE1 7RU. \\ (abdullah.alhasant@ncl.ac.uk; bayan.sharif@ncl.ac.uk; charalampos.tsimenidis@ncl.ac.uk; \\ s.boussakta@newcastle.ac.uk)
}

\begin{abstract}
In this paper, a collaborative localization technique based on the Direction of Estimation (DoE) is proposed. Two directions, Border-to-centre B-C and Centre-to-Border $C-B$, are investigated and their location accuracies are evaluated. The results show that the $B-C$ method approach outperforms the $C-B$ method based on the Root-Mean Square Error (RMSE) and Cramér-Rao Bound (CRB). The new B-C DoE method is demonstrated using different deployment strategies and node densities. The simulation results show that the B-C DoE algorithm decreases the accumulated errors of location estimation approximately by $12 \%$ over conventional collaborative algorithms; in addition its performance is clearly superior to the performance of other DoE methods.
\end{abstract}

Keywords - Cramér-Rao Bound, Direction of Estimation, Error Accumulation, Localization System, Received Signal Strength, Sensor Deployment.

\section{INTRODUCTION}

Recent technological advancement in radio frequencies (RF) and Integrated Circuits (IC) has led to the manufacturing of low power electronic devices embedded with on-board processing, storage, wireless communication and sensing capabilities, such embedded devices are called Wireless Sensor Network (WSN). WSN without localization algorithm are often considered meaningless [1]-[5], for example in wild animal's behaviour, enemy tracking and logistics, they will become out of control and will fail monitoring [6]. Furthermore, as the localization algorithm is application-dependant, it is sometimes also used to reduce the complexity of the routing algorithm, since if each sensor knows the location of its neighbour; it can then decide what the next hop is.

In large-scale networks with thousands of very small and battery-powered sensor nodes, accurate and low-cost sensor localization is a critical problem [7]. Global Positioning Systems GPS are currently relatively expensive, as they require that every node should be equipped with GPS. Furthermore, they are unsuitable for indoor environment due to NLOS (non-line of sight) [1].

Three main range-based techniques have been studied for WSN, including time-of-arrival (TOA), angle-of-arrival (AOA), and received signal strength (RSS), each of which has its advantages and limitations. Although TOA and AOA algorithms produce more accurate location estimation, they are expensive solutions due to synchronization and hardware requirements [8].

RSS measurements are unreliable due to the fact that RSS is largely affected by signal multi-path fading and shadowing problems. However, RSS provides the cheapest and simplest technology as no extra hardware is needed such as the case in TOA and AOA [2]. In addition, the RSS of RF signals can easily be measured in almost all existing wireless systems [3]. Trilateration and Multilateration algorithms can be used for RSS-based localization systems. Although these algorithms are very simple in computation, they suffer from the requirement of a minimum number of reference nodes to be used, position of these reference nodes and inaccurate position estimation [8], [9].

Recently, collaborative algorithms have become the most interesting RSS-based localization techniques. The collaboration of more unknown-location sensor nodes can significantly improve the performance of localization algorithms; even if unreliable RSS measurements are used, which is proven in [4]. This paper endeavours to estimate the sensor location using RSS-based collaborative techniques. New Direction of Estimation (DoE) algorithm is proposed, and its performance is analyzed and validated. Throughout this research, in addition to Root Mean Square Error (RMSE), the Cramér-Rao Bounds (CRB) will also be considered.

The rest of this paper is presented as follows: we begin in section II by exploring the related works. The problem is formulated in section III, and the proposed DoE method is explained in Section IV. The performance evaluation and various simulation scenarios are presented in section $\mathrm{V}$ and VI respectively. Finally conclusions are drawn in section VII.

\section{RELATED WORK}

Collaborative localization techniques are a class of technique that utilize distance measurements between pairs of location-unaware sensor nodes [6]. It allows the locationunaware sensor node that is not in the range of any reference node to be located, using one of its known-location neighbours. Several collaborative localization techniques have been proposed for sensor networks such as Maximum 
Likelihood Estimation (MLE) [4], Multi-dimensional Scaling (MDS) [2], [4], [5] and MDS-MLE [3], [6], [7].

MLE determines the parameters that maximize the probability (likelihood) of sample data (statistical data), and in this approach, the system accuracy improves as the node density increases [4]. On the other hand, MDS produces a relative map that shows how the sensor nodes are aligned with respect to each other in 2D or 3D space [2]. In order, to gain benefit from both MLE and MDS, an integrated algorithm called MDS-MLE has been proposed [3]. The simulation results in [3] show that the combined MDS-MLE outperforms the MDS or MLE algorithms.

In general, the collaborative localization algorithm can be either iterative or non-iterative. In non-iterative (atomic localization), all nodes estimate their position concurrently and only in one iteration, which is fast and fully distributed, however, non-iterative algorithms are impractical for large scale sensor networks. While in iterative methods such as DPE [8] and AHLos [9], once the node estimates its position, it acts as a reference node for its neighbours, increasing the system coverage. Whereas, in addition to the $n$ iterations needed for $n$ sensors to be estimated, it suffers from the drawback of error accumulation.

In contrast to conventional iterative localization, the higher density of unknown nodes in the iterative collaborative algorithm actually increases the accuracy of the location estimation [10]. Hence, in this paper, the iterative collaborative algorithm will be investigated. It will be concerned with reducing the error propagation inherent in the iterative process.

\section{PRoBlem StATEMENT}

The Received Signal Strength (RSS) is the signal power loss along the path from transmitter to receiver. In free space, the power of the received signal decays proportional to $d^{-2}$, where $d$ is the Transmitter-Receiver distance [11]. More generally and for realistic channels, the received power $P_{i j}$ at sensor $j$ transmitted by sensor $i$ defined as

$$
P_{i j}=P_{0}-n_{p} \times 10 \log _{10} \frac{d_{i j}}{d_{0}}
$$

where $P_{0}$ is received power measured in Decibel milliwatts $(\mathrm{dBm})$ at reference distance $d_{0}$ (usually $d_{0}=1 \mathrm{~m}$ ), $d_{i j}$ is the distance between sensor $i$ and sensor $j$, and $n_{p}$ is the path loss exponent (i.e. for a free space model $n_{p}=2$ ).

As mentioned in the introduction, two environmentdependant sources significantly affect the measured power; multipath propagation and shadowing. Multipath signals are often known as frequency-selective fading problem, which are caused by multiple signals with different amplitudes and phases arrive at receiver, these signals add constructively and destructively as a function of frequency. The effects of this error can be diminished by using a spread-spectrum method [7]. In our research, we remove the effects of this fading by computing the average of multiple RSSs at the receiver from the same transmitter [7].

In shadowing, the signal is attenuated due to obstructions (furniture, walls, and other objects), and this attenuation randomly affects the signal, which makes it difficult to estimate the distance. In order to deal with the randomness of shadowing, the Gaussian random variable $X_{\sigma} \sim\left(0, \sigma^{2}{ }_{d B}\right)$ (with zero mean and variance $\sigma_{d B}$ depends on the environment) should be added to the received power $P_{i j}$ in (1) [12]. So, we can write the RSS as

$$
r s s_{i j}=P_{i j}+X_{\sigma}
$$

The received signal strength RSS is usually considered symmetric [2], i.e. $\mathrm{RSS}_{\mathrm{ij}}=\mathrm{RSS}_{\mathrm{ji}}$, for $i \neq j$. Given (1) and (2), [4].

Assuming that a sensor network consists of $m$ unknown sensor nodes and $n$ reference nodes, then the problem of relative localization corresponds to the estimation of the vector parameters $Z=\left[z_{1}, z_{2}, \ldots, z_{m}\right]$, where $1 \leq i \leq m$, and $z_{i}$ is the device parameter $\left[x_{i}, y_{i}\right]$ for two dimensional systems. The likelihood function of $Z$ that most likely results in a given value of $R S S$ is

$$
f_{R \mid Z}(R S S \mid Z)=\frac{1}{\sqrt{2 \pi \sigma^{2}}} \exp \left(-\frac{\left(r s s_{i j}-p_{i j}\right)^{2}}{2 \sigma^{2}}\right)
$$

The Maximum Likelihood Estimation of the distance between sensor $i$ and $j\left(\hat{d}_{i j}\right)$ is obtained by differentiating the $\log$ likelihood in (3) with respect to $d$ and setting the result equal to zero [13]:

$$
\left.\frac{\partial \log f_{R \mid Z}(R \mid Z)}{\partial Z}\right|_{d=d_{m l}}=0
$$

The MLE of $\hat{d}_{m l}$ is the value of $d$ at which the likelihood function is a maximum. Thus

$$
\hat{d}_{m l_{i j}}=d_{0} 10^{\frac{P_{0}-R S s_{i j}}{10 n_{p}}}
$$

The vector parameters $\left[Z_{x} Z_{y}\right]^{T}$ for $i=1, \ldots, m+n$ and $j=1, \ldots, m+n$, can be estimated using the cost minimization function as follows

$$
\hat{Z}=\arg _{\left\{x_{i}, y_{i}\right\}} \min \left\{\sum_{i=1}^{m+n} \sum_{\substack{j<i \\ j \in H(i)}}\left(\ln \frac{\hat{d}_{i j}{ }^{2}}{d^{2}\left(\check{\mathbf{z}}_{i}, \check{\mathbf{z}}_{j}\right)}\right)\right\}
$$

where $\hat{Z}$ is a vector of estimated device parameters defined as $\hat{Z}=\left[\hat{z}_{1}, \hat{z}_{2}, \ldots, \hat{z}_{m}\right], H(i)=\{j$ : device $j$ makes pair-wise observation with device $i\}$ (in our case, we assume an infinite transmission range, i.e. $H(i)=\{j: 1 \leq j \leq n+$ $m$ and $i \neq j\}$ ), and

$$
d\left(\check{\mathbf{z}}_{\boldsymbol{i}}, \check{\mathbf{z}}_{\boldsymbol{j}}\right)=\sqrt{\left(\check{x}_{i}-\check{x}_{j}\right)^{2}+\left(\check{y}_{i}-\check{y}_{j}\right)^{2}}
$$

where $\left(\check{x}_{i}, \breve{y}_{i}\right)$ represents the initial estimate of the location for sensor $i$. 


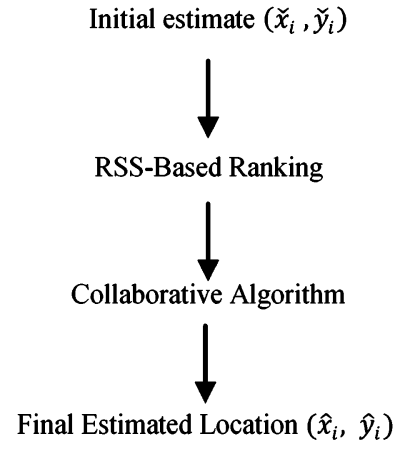

Fig.1. Direction of Estimation (DoE) algorithm, $\left(\check{x}_{i}, \check{y}_{i}\right)$ is the initial estimate and $\left(\hat{x}_{i}, \hat{v}_{i}\right)$ is the final estimate for $1<i<m$.

\section{DIRECTION OF ESTIMATION (DOE)}

The new proposed technique focuses on reducing the total error accumulation resulting from the iterative collaborative algorithm. Usually, the collaborative algorithms are started by randomly guessing an initial estimate for each unknown node. Based on the Direction of Estimation (DoE), we propose a new method, referred to as DoE, to improve the location accuracy over traditional collaborative methods.

Instead of starting the estimation randomly, starting from the more reliable border nodes towards the centre nodes should reduce the total accumulated error. In order to start the estimation process from border to centre (B-C), we should rank the nodes based on their RSS connectivity from reference nodes. Figure 1 illustrates the DoE algorithm, where $m$ is number of unknown nodes, $\left(\hat{x}_{i}, \hat{y}_{i}\right)$ is the final estimate for $1 \leq i \leq m$, and $\left(\check{x}_{i}, \breve{y}_{i}\right)$ was explained in the previous section.

The RSS-based ranking can be achieved using many techniques. Initially, after applying the normal collaborative localization algorithm, we use the estimated locations to obtain the new order, and then the location effectiveness for each node from the centre of the deployment area is computed. The node that has the highest effectiveness will be chosen to start the estimation.

To compute the location effectiveness, we consider $m$ unknown nodes within a deployment area $(k \times l)$ that has a diameter $r$. The location effectiveness ( $e f f$ ) of sensor $i$ is defined as:

$$
e f f(i)=\frac{\sqrt{\left(\check{x}_{i}-x_{c}\right)^{2}+\left(\check{y}_{i}-y_{c}\right)^{2}}}{r / 2} \times 100 \%
$$

where $1 \leq i \leq m$, and $\left(x_{c}, y_{c}\right)$ is the central point of the deployment area (if the origin point is $(0,0)$, then $x_{c}=\frac{k}{2}$ and $\left.y_{c}=\frac{l}{2}\right)$. Once we have the location effectiveness for each unknown sensor node, a selection sort algorithm [14] can be used. Finally, the MLE discussed in the previous section can be applied for the new orders of the initial estimates. In the next section the performance of the new DoE method is investigated.

\section{PERformance Evaluation}

Basically, the most used method to calculate the accuracy of the estimator in localization algorithms is the RMSE. The Location error of the new estimated location is defined as the Euclidean distance between the location estimate $(\hat{x}, \hat{y})$ and the actual location of the unknown node $(x, y)$, so, if the number of unknown sensors is $m$, the RMSE will be

$$
R M S E=\sqrt{\frac{1}{m} \sum_{i=1}^{m}\left\{\left(\hat{x}_{i}-x_{i}\right)^{2}+\left(\hat{y}_{i}-y_{i}\right)^{2}\right\}}
$$

Furthermore, we define the performance of DoE method with reference to the Cramér-Rao Bound (CRB). The CRB provides the lower bound on the variance achievable by particular unbiased location estimator [15]. The CRB of the unbiased RSS-based is derived in [7]. Some bias in the estimator might be tolerated to reduce the variance, in such cases, the bound can be adapted [16].

If we denote $\operatorname{Cov}(\hat{Z})$ as the covariance matrix of the unbiased estimator, then any unbiased estimator $(\hat{Z})$ must satisfy

$$
\operatorname{Cov}(\hat{Z}) \geq F^{-1}(Z)
$$

where $\mathrm{F}^{-1}(Z)$ is the Fisher Information Matrix FIM [7]. Therefore, specifically for each node $i$ where $i=1, \ldots, m$, the bound in (10) becomes

$$
\begin{aligned}
\operatorname{Var}_{Z}\left(\hat{x}_{i}\right)+\operatorname{Var}_{Z} & \left(\hat{y}_{i}\right) \\
& \geq\left\{F^{-1}(Z)\right\}_{i i}+\left\{F^{-1}(Z)\right\}_{(m+i)(m+i)}
\end{aligned}
$$

Dividing both sides by $m$, taking the square root, and comparing the result with (9), we then have

$$
\operatorname{RMSE}(\hat{Z}) \geq \operatorname{RMS}\left(F^{-1}(Z)\right)
$$

and

$$
R M S\left(F^{-1}(Z)\right)=\sqrt{\frac{1}{m} \sum_{i=1}^{2 m}\left\{F^{-1}(Z)\right\}_{i i}}
$$

where $R M S\left(F^{-1}(Z)\right)$ is the RMS of the localization bound.

The numerical example in figure 2 was used to study the CRB lower bound in a grid deployment. Assuming that the number of grid point per side is $G$, and then we have $G \times G$ nodes. If the number of reference nodes is $n$, then the number of unknown nodes is equal to $G \times G-n$. In our particular example, we use $G=6$, and $n=4$, so $m=32$ nodes in all cases.

The CRB depends on the number of unknown nodes $(m)$, reference nodes $(n)$, and $\sigma / n_{p}$ ratio [13]. As shown in figure $2 \mathrm{a}$, the lower bound rapidly decreases as the number of unknown nodes is increased, while, as shown in figure $2 b$, it slightly decreases as the number of reference nodes is 


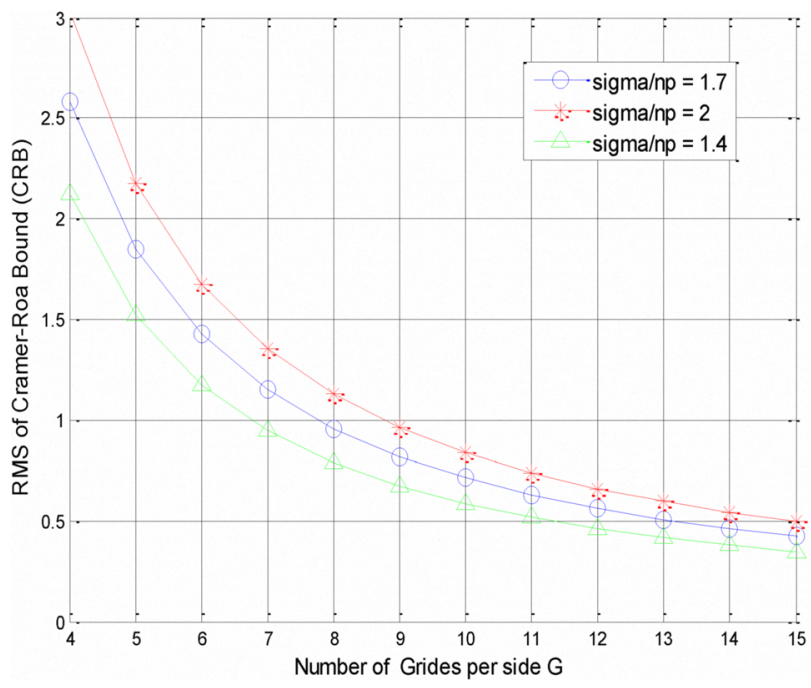

(a)

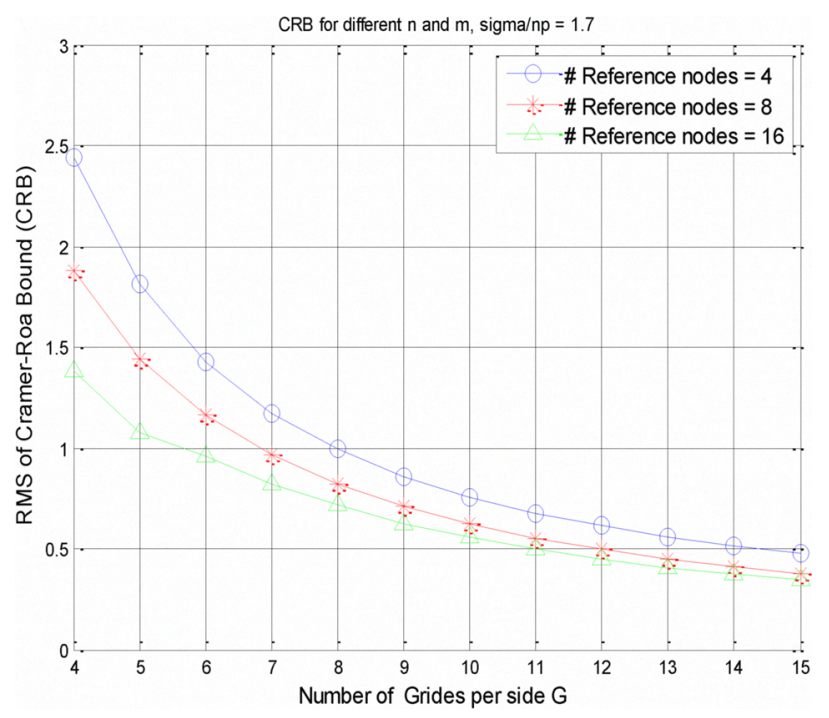

(b)

Fig.2. CRB lower bound with respect to (a) the number of unknown nodes, (b) the number of reference nodes.

increased. Meanwhile, the lower bound CRB is directly increased when the $\sigma / n_{p}$ ratio is increased.

\section{SiMULATION RESULTS}

The performance of the DoE algorithm is investigated through an extensive simulation study. The impact of several factors on the DoE collaborative localization including unknown nodes density, reference nodes density, direction of estimation (C-B and B-C), and unknown nodes deployment strategies (random and grid) is studied in detail. The theoretical lower bound (CRB) is also given for each case.

In our scenarios, the four reference devices are positioned at the four corners of an $20 m \times 20 m$ area. The unknown nodes are generated randomly. The estimated distance is computed based on the equation in (5). Thereafter, the minimization problem in (6) is solved and implemented using
Table 1. Typical Values and Meanings for Different Simulation Parameters

\begin{tabular}{clc}
\hline \hline \multirow{2}{*}{ Parameter } & \multicolumn{1}{c}{ Meaning } & Typical value \\
\hline$p_{t}$ & Transmitting power & 0.001 Watts \\
$d_{0}$ & Reference Distance & $1 \mathrm{~m}$ \\
$F_{c}$ & Carrier Frequency & $2443 \mathrm{MHz}$ \\
$n_{p}$ & Path Loss Exponent & $2.3[4]$ \\
$K \times l$ & Area of deployment & $20 \mathrm{~m} \times 20 \mathrm{~m}$ \\
& & \\
\hline \hline
\end{tabular}

the iterative numerical optimization tools in MATLAB. Table 1 summarizes the typical values and meaning of the environmental parameters used in our simulation. Figure 3 plots the ideal channel model and the simulated channel model based on the same values shown in table 1 .

One of the most important questions is how the iterative and non-iterative algorithms differ in collaborative localization systems. We showed in figure 4 , that they behave conversely with respect to the density of the unknown node. When the iterative algorithm is used, and as the unknown node density increases, then the RMSE is decreased, while it increased in the case of the non-iterative algorithm.

The performance of the normal DoE, C-B DoE and B-C DoE methods with respect to unknown node density and reference node density are plotted in figure 5. It is apparent in figure $5 \mathrm{a}$, that the $\mathrm{B}-\mathrm{C}$ DoE outperforms the others techniques in term of unknown node density. On average, the RMSE of the location estimation is reduced by approximately $12 \%$. Figure $5 \mathrm{~b}$ demonstrates that as the reference nodes density increases, the RMSE of the C-B DoE algorithm is decreased, however, the B-C DoE algorithm consistently achieves a lower RMSE. Moreover, all three DoE algorithms most likely perform in the same way in the case of a very large number of reference nodes, which is not a recommended situation, in other words, the $\mathrm{B}-\mathrm{C}$ DoE algorithm becomes quite useful as the ratio of the number of unknown nodes to the number of reference nodes increases.

In the previous results, the unknown nodes are randomly deployed in the sensor field area. In the following, the proposed techniques were tested and simulated over a grid deployment. We used the same values of the parameter illustrated in table 1 . The results in figure 6a give the RMSE of all DoE methods with regard to the number of unknown nodes. As with the random deployment, in a grid deployment, the B-C DoE is shown to improve the accuracy of location estimations and is slightly better than the normal DoE algorithm. In the same way, figure $6 \mathrm{~b}$ shows the relationship between the DoE algorithms and the density of reference nodes. Although the performance of the DoE algorithms improves with the increase in the number of reference nodes (up 12 nodes), however, a further increase in the number of reference nodes will only result in a marginal improvement. This result is also confirmed by the CRB bound in figure $6 \mathrm{~b}$. 


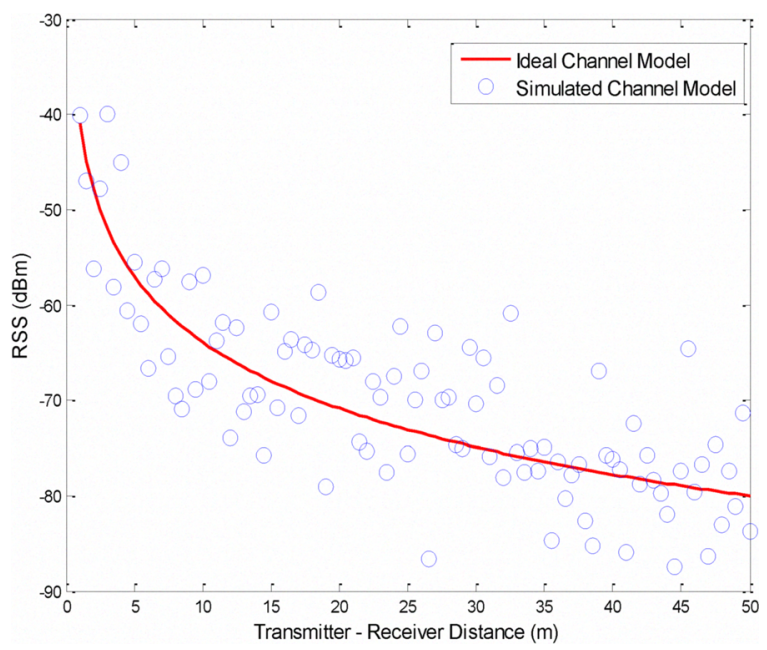

Fig.3. Plot of Ideal channel model and simulated channel model.

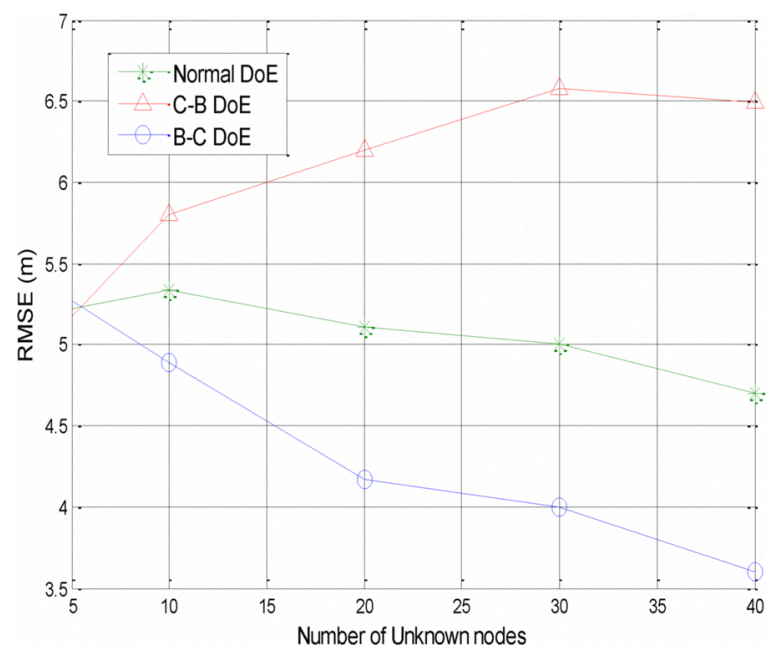

(a)

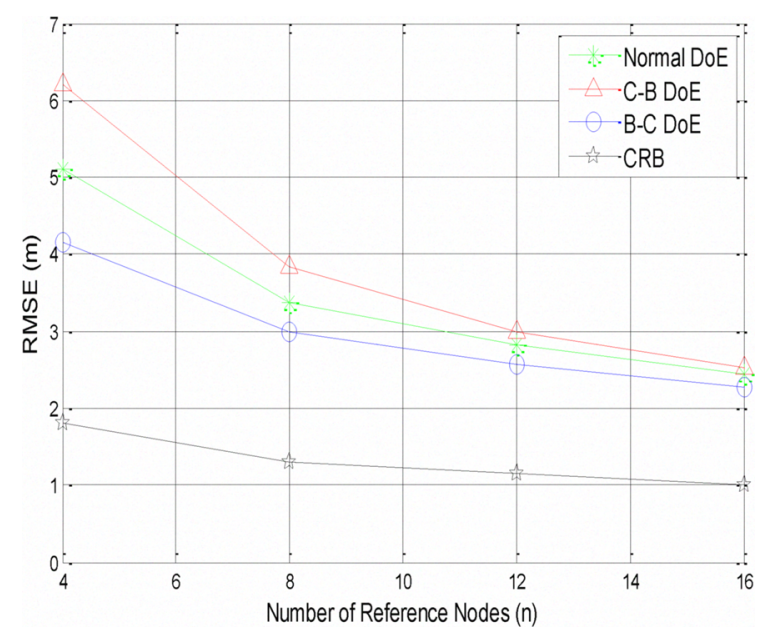

(b)

Fig.5. Performance of DoE algorithm (in random deployment) with respect to (a) the number of unknown nodes $n=4$, (b) the number of reference nodes and $\mathrm{m}=20$.

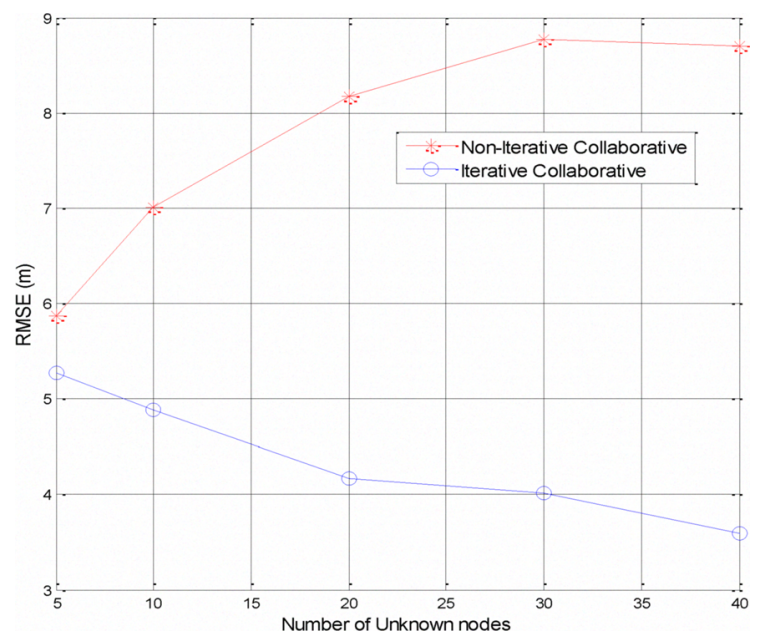

Fig.4. Iterative and non-iterative collaborative, with different unknown nodes $(m)$ and four reference nodes $(n=4)$.

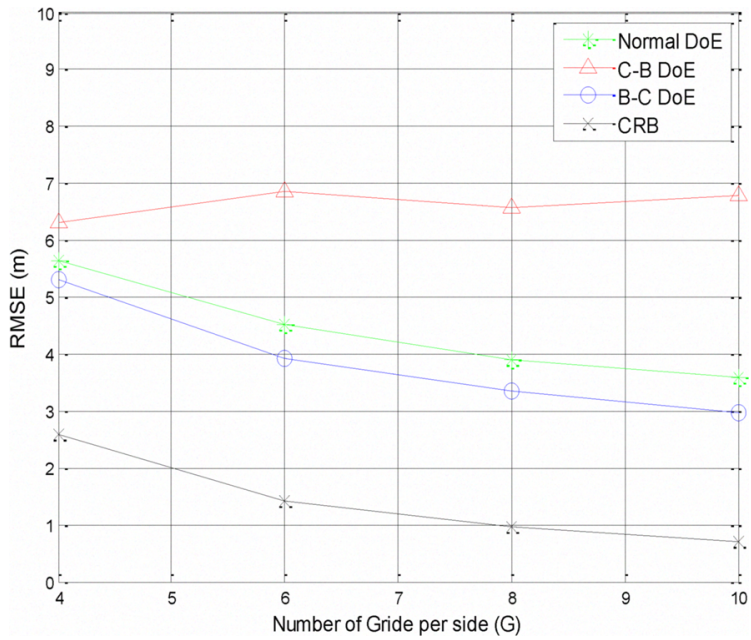

(a)

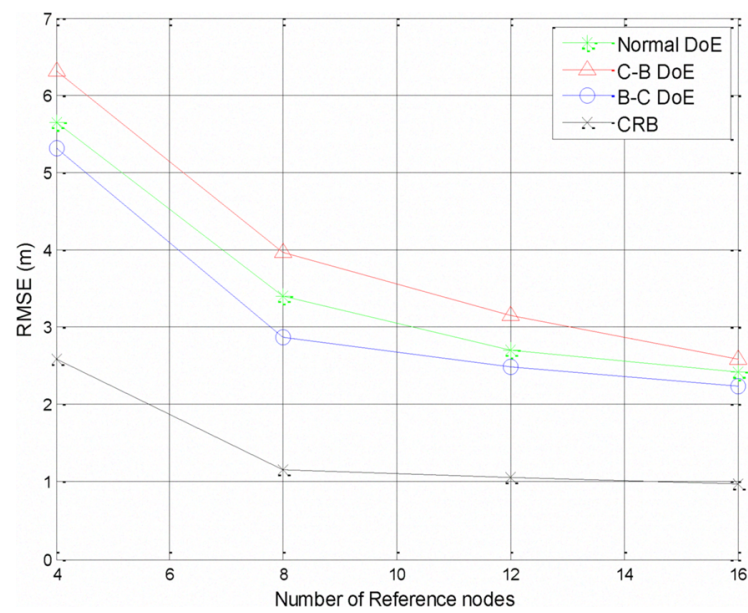

(b)

Fig.6. Performance of DoE algorithm (in Grid deployment) with respect to (a) the number of unknown nodes, $n=4$, (b) the number of reference nodes $\mathrm{G}=6$. 


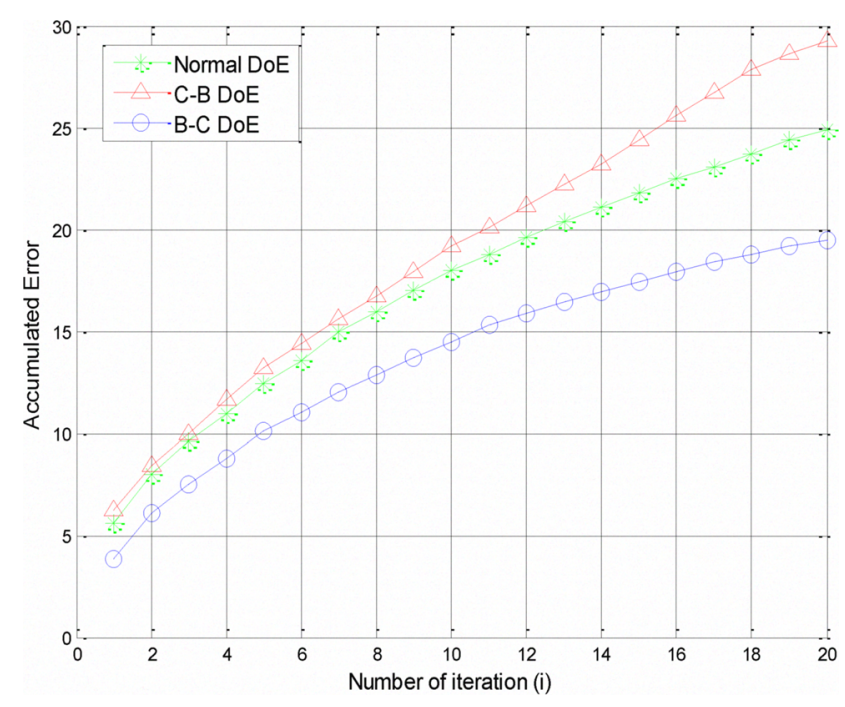

Fig.7. Accumulated Errors for each iteration in the DoE algorithms, where $m=20$ and $n=4$.

Finally, the accumulated errors for each iteration in all DoE methods are shown in figure 7. It is evident that for $m=20$ nodes (i.e. 20 iterations), the B-C DoE algorithm provides lower accumulated errors for all iterations. Furthermore, the progressive improvement in accumulated errors exhibited by the B-C DoE is shown to be significant as the number of iterations is increased.

\section{CONCLUSION AND FUTURE WORK}

The aim of the work presented in this paper is to reduce the error accumulation in iterative collaborative location estimation. An approach based on Direction of Estimation was proposed and assessed through simulations for different scenarios and deployment strategies.

The simulation results have shown that the B-C DoE algorithm outperforms other methods with respect to both unknown nodes and reference nodes densities. The B-C DoE was also demonstrated to reduce error accumulation and provide performance gains in random and grid nodes deployments.

Although it has been shown that the B-C DoE improves the location estimation, there is still a large gap between the theoretical lower bound CRB and the result produced by the B-C DoE algorithm. Consequently, the DoE method can be modified and improved using specific RSS ranking techniques. In addition, the B-C DoE should be implemented using a distributed localization algorithm, which can be accomplished using RSS pair-wise ranking method.

\section{ACKNOWLEDGMENT}

The authors would like to thank AL-HUSSIEN BIN TALAL UNIVERSITY - JORDAN, for their financial support of Mr. Alhasanat's research studies.

\section{REFERENCES}

[1] M. Sugano, T. Kawazoe, Y. Ohta, and M. Murata, "Indoor localization system using RSSI measurement of wireless sensor network based on ZigBee standard," in Proceedings of the IASTED International Conference on WIRELESS NETWORKS AND EMERGING TECHNOLOGIES, Part of the Sixth IASTED International MultiConference on WIRELESS AND OPTICAL COMMUNICATIONS, 2006.

[2] X. Ji and H. Zha, "Sensor positioning in wireless ad-hoc sensor networks using multidimensional scaling," in Proceedings - IEEE INFOCOM, 2004, pp. 2652-2661.

[3] X. Li, "Collaborative localization with received-signal strength in wireless sensor networks," IEEE Transactions on Vehicular Technology, vol. 56, pp. 3807-3817, 2007.

[4] N. Patwari, A. O. Hero Iii, M. Perkins, N. S. Correal, and R. J. O'Dea, "Relative location estimation in wireless sensor networks," IEEE Transactions on Signal Processing, vol. 51, pp. 2137-2148, 2003.

[5] Y. Shang, W. Ruml, Y. Zhang, and M. Fromherz, "Localization from connectivity in sensor networks," IEEE Transactions on Parallel and Distributed Systems, vol. 15, pp. 961-974, 2004.

[6] B. T. Avanthi Koneru, "COMPARATIVE STUDY OF RSS-BASED COLLABORATIVE LOCALIZATION METHODS IN WIRELESS SENSOR NETWORKS," in Department of Computer Science and Engineering. vol. MASTER OF SCIENCE: UNIVERSITY OF NORTH TEXAS, December 2006.

[7] N. Patwari, "LOCATION ESTIMATION IN SENSOR NETWORK," in Electrical Engineering: Systems. vol. PhD: The University of Michigan, 2005.

[8] H. A. B. F. De Oliveira, E. F. Nakamura, A. A. F. Loureiro, and A. Boukerche, "Directed position estimation: A recursive localization approach for wireless sensor networks," in Proceedings - International Conference on Computer Communications and Networks, ICCCN, 2005, pp. 557-562.

[9] A. Savvides, C. C. Han, and M. B. Strivastava, "Dynamic fine-grained localization in ad-hoc networks of sensors," in Proceedings of the Annual International Conference on Mobile Computing and Networking, MOBICOM, 2001, pp. 166-179.

[10] R. J. O. D. N. Patwari, and Y. Wang, "Relative Location in wireless networks " IEEE VTC, vol. 2, pp. 1149-1153, May 2001.

[11] T. Rappaport, Wireless Communications: Principles and Practice, 2nd ed.: Prentice Hall PTR.

[12] K. Pahlavan, P. Krishnamurthy, and J. Beneat, "Wideband radio propagation modeling for indoor geolocation applications," IEEE Communications Magazine, vol. 36, pp. 60-65, 1998.

[13] H. L. V. TREES, Detection, Estimation, and Modulation Theory, Part I: John Wiley \& Sons, 1968.

[14] H. M. Mahmoud, Sorting: a distribution theory, illustrated ed.: WileyIEEE, 2000.

[15] N. Patwari, J. N. Ash, S. Kyperountas, and A. O. Hero Iii, "Cooperative localization in wireless sensor networks," IEEE SIGNAL PROCESSING MAGAZINE, vol. 54, 2005.

[16] A. O. Hero Iii, J. A. Fessler, and M. Usman, "Exploring estimator biasvariance tradeoffs using the uniform CR bound," IEEE Transactions on Signal Processing, vol. 44, pp. 2026-2041, 1996. 